\title{
Response for light scattered in the ocular fundus from double-pass and Hartmann-Shack estimations
}

\author{
Carlos E. García-Guerra ${ }^{1,}{ }^{*}$, Mikel Aldaba $^{1}$, Montserrat Arjona ${ }^{1}$, Fernando \\ Díaz-Doutón ${ }^{1}$, Joan A. Martínez-Roda ${ }^{1}$, And Jaume Pujol ${ }^{1}$
}

\begin{abstract}
Double-pass (DP) and Hartmann-Shack (HS) are complementary techniques based on reflections of light in the ocular fundus that may be used to estimate the optical properties of the human eye. Under conventional data processing, both of these assessment modes provide information on aberrations. In addition, DP data contain the effects of scattering. In the ocular fundus, this phenomenon may arise from the interaction of light with not only the retina, but also deeper layers up to which certain wavelengths may penetrate. In this work, we estimate the response of the ocular fundus to incident light by fitting the deviations between DP and HS estimations using an exponential model. In measurements with negligible intraocular scattering, such differences may be related to the lateral spreading of light that occurs in the ocular fundus due to the diffusive properties of the media at the working wavelength. The proposed model was applied in young healthy eyes to evaluate the performance of scattering in such population. Besides giving a parameter with information on the ocular fundus, the model contributes to the understanding of the differences between DP and HS estimations.
\end{abstract}

http://dx.doi.org/10.1364/ao.XX.XXXXXX

\section{INTRODUCTION}

Double-pass (DP) instruments [1] and Hartmann-Shack (HS) aberrometers [2] are systems that rely on the projection of a point source on the ocular fundus to estimate optical quality descriptors. Both of these assessment modes provide information on aberrations [3]. Under conventional data analysis, only those descriptors derived from DP data contain the effects of scattering $[3,4]$. In the case of the eye, such phenomenon may arise from the interaction of light with any structure with diffusive properties involved in the process [5].

When light interacts with the ocular fundus, it suffers from absorption, reflections, and scattering [6]. In the case of the retina, the light is waveguided within photoreceptors and part of the incident energy is reflected by the pigment epithelium towards the pupil with a given angular distribution and variety of phases [7]. The part of the incident light that is not reflected or absorbed by the pigment epithelium may penetrate up to the choroid, where the presence of blood disperses light laterally [6]. It has been suggested that light emitted towards the pupil from this layer is the result of forward scattering and reflections at interfaces such as vessels and sclera. This situation is more evident at certain wavelengths $[8,9]$ in function of the absorption properties of the pigment epithelium [6].

The scattering process occurring in the ocular fundus has been considered in visual optics to explain differences between DP data and that obtained with instruments that are unaffected by scattering, such as interferometric devices [5] and HS sensors [10]. These works suggest that the differences may arise from light scattered in the choroid. On the other hand, it has been also corroborated that variations in both the structure of the ocular fundus [11, 12] and the wavelength [13] influence the response of DP instruments. Some authors have developed theoretical models based on approximations of experimental data that predict the response of the ocular fundus to incident light at different wavelengths [6]. However, this knowledge is usually not incorporated into the DP formulation, maybe because it results difficult to separate its contribution to the DP response. In addition, it is not clear if such scattered light contributes to the image formation process that occurs in the retina [5].

In a previous work, we presented an open-view system that combines a DP instrument and a HS sensor [12]. During the calibration process, it was demonstrated that these assessment modes provide comparable estimations when the influence of 
the ocular fundus is obviated. Despite this, DP curves deviates from HS ones in measurements on real eyes. As other authors $[5,10]$, we suggested that such deviations may be provoked by the scattering properties of the ocular fundus.

The objective of this article is to determine the response of the ocular fundus of healthy young population in infrared light. The deviations between comparable DP and HS estimations are used for this purpose. The differences between curves are fitted using an exponential model that accounts for the modulation transfer function (MTF) of the scattering process and gives information on the lateral spreading of light that occurs in the ocular fundus. The model was applied to measurements on real eyes unaffected by intraocular scattering. In this manner, the differences between curves may be attributed to effects produced in the ocular fundus at the working wavelength. We consider that the proposed model provides a better understanding on the processes occurring in the ocular fundus in DP measurements. This knowledge could be then used to improve the accuracy of the parameters with information on the optical quality of the eye, such as the MTF, Strehl ratio, scatter indexes, among others. In addition, the model could be also incorporated into either HS or DP curves to provide comparable estimations between techniques. On the other hand, the experimental data presented here may be used as reference to detect deviations with respect to average values as an indicator of possible problems in the choroid. Further studies may be carried on to evaluate the correlation between scattering in the ocular fundus and visual performance.

This article presents first the proposed model by incorporating the effects of light scattered in the ocular fundus into the DP formulation. Once defined, the model is used to describe the differences between DP and HS data in estimations describing the optical properties of real eyes. Finally, general conclusions are presented at the end of this work.

\section{MODEL FOR LIGHT SCATTERED IN THE OCULAR FUNDUS}

The DP technique is a useful tool to describe the optical properties of the eye under a variety of conditions [14-16]. In this, first a point source is projected on the retina; there the light suffers a time-varying complex reflection process and then the aerial retinal image is recorded after the second-pass of light through the optics of the eye [1]. A theoretical description of this process is presented in [17]. During the development of the expressions, the reflection process occurring in the fundus is assumed to be $\delta$-correlated [18]. Under this consideration, the dependence on the ocular fundus is eliminated for longexposure (incoherent) DP images. However, models for the response of the fundus to incident light [6] suggest that the scattering arising from the iteration of light with the fundus is governed by correlation functions different than $\delta$.

Hodgkinson et al. introduced a mathematical description of the response of the ocular fundus to incident light based on measurements of an artificial model emulating the properties of the choroid [6]. In summary, the results of the authors predict the PSF of the ocular fundus at different wavelengths. In systems without scattering, this response is given by a $\delta$ function. However, the application of the model indicates not only that the PSF may be fitted using other type of functions, but also that the standard deviation of the response (the lateral spreading of light) is proportional to the thickness of the layers containing blood cells, which in the case of the eye refer to the choroid.

The objective of this section is to obtain a model that accounts for the effects of the scattering process occurring in layers of the ocular fundus beyond the retina. In this context, it is necessary to modify the shape of the correlation function in the expression developed in [17] to account for the effects of scattering in DP images. The theoretical development is presented in detail until obtaining an expression for the MTF that considers the effects of scattering from the ocular fundus.

\section{A. The DP expression}

Consider the image-formation process in the DP technique described by Artal et al. [17]. From there, it is known that the instantaneous (coherent) intensity of the double-pass process computed as the square modulus of the amplitude field $U_{i}^{\prime \prime}$ at the second pass image plane $\left(x^{\prime \prime}, y^{\prime \prime}\right)$ is expressed as,

$$
\begin{aligned}
I_{i}^{\prime \prime}\left(x^{\prime \prime}, y^{\prime \prime}\right)= & \left|U_{i}^{\prime \prime}\left(x^{\prime \prime}, y^{\prime \prime}\right)\right|^{2} \\
= & m^{4} \mid h_{2}\left(m x^{\prime \prime}, m y^{\prime \prime}\right) \otimes \\
& {\left.\left[h_{1}\left(-m x^{\prime \prime},-m y^{\prime \prime}\right) R_{i}\left(-m x^{\prime \prime},-m y^{\prime \prime}\right)\right]\right|^{2} }
\end{aligned}
$$

where $m$ is the modulus of the first pass magnification, $h_{1}$ and $h_{2}$ are the first and second pass amplitude-spread functions, and $\otimes$ denotes convolution. As originally conceived, $R_{i}$ accounts for the instantaneous reflection factor of the retina. However, there are cases in which deeper layers of the ocular fundus should be considered to obtain a more realistic representation of the DP process, especially under the presence of scattering. Therefore, the term $R_{i}$ as used here incorporates the effects arising in any of the layers of the ocular fundus.

Let consider hereafter the unidimensional case for the sake of clarity. The incoherent DP response is obtained by averaging a number of the coherent estimations denoted by equation 1 [17]. The image resulting from this operation is known to be linear in intensity [19]. In this manner, the square magnitudes affecting the intensity can be applied independently on each side of the convolution and the results can be expressed in terms of its intensity PSF. Assuming that the spread functions are deterministic, the intensity can be written in the following form,

$$
\begin{aligned}
\left\langle I_{i}^{\prime \prime}\left(x^{\prime \prime}\right)\right\rangle= & I^{\prime \prime}\left(x^{\prime \prime}\right) \\
= & P S F_{2}\left(m x^{\prime \prime}\right) \otimes \\
& {\left[P S F_{1}\left(-m x^{\prime \prime}\right)\left\langle R_{i}\left(-m x^{\prime \prime}\right) R_{i}^{*}\left(-m x_{1}^{\prime \prime}\right)\right\rangle\right] }
\end{aligned}
$$

The term $\left\langle R_{i} R_{i}^{*}\right\rangle$ represents the realization of the ensemble average between the scattered fields at two point positions $-m x^{\prime \prime}$ and $-m x_{1}^{\prime \prime}[20]$ and is computed as an autocorrelation function. Moreover, the ensemble average may be substituted by a space average because the process is considered as statistically stationary [21]. The result of this operation is a function that depends on the properties of the rough surface (ocular fundus).

\section{B. Modeling scattering as lateral spreading of light}

In the incoherent double-pass process given by equation 2 , the light resulting from the interaction of the incident energy with the ocular fundus is expressed as a multiplication between the first pass PSF and the long-exposure reflection factor of the 
surface. Let introduce in the model the concept of PSF of the ocular fundus. This is denoted as $P S F_{F}$ and represents the response of the fundus when this is impinged by a ray. Mathematically, the contribution of all the rays to the response is given by the convolution [19]. If the multiplicative reflection process is substituted by a convolution between the incident light and $P S F_{F}$, the double-pass process may be expresses as,

$$
I^{\prime \prime}\left(x^{\prime \prime}\right)=P S F_{2}\left(m x^{\prime \prime}\right) \otimes\left[P S F_{1}\left(-m x^{\prime \prime}\right) \otimes P S F_{F}\left(-m x^{\prime \prime}\right)\right]
$$

It results convenient to express last expression in the Fourier domain, where the convolutions can be evaluated as multiplications between the spectral representations of the functions involved in the process [22]. The modulus of the Fourier transformed PSF leads to the MTF after proper normalization [19]. In this manner, the spectral representation of the DP image can be mathematically represented in terms of MTFs accounting for the responses of not only the first and second pass, but also the fundus,

$$
\begin{aligned}
\operatorname{MTF}(u) & \propto\left|\mathcal{F}\left\{I^{\prime \prime}\left(x^{\prime \prime}\right)\right\}\right| \\
& =\operatorname{MTF}_{2}(u) \operatorname{MTF}_{1}(u) \operatorname{MTF}_{F}(u)
\end{aligned}
$$

$M T F_{F}$ should be specified to continue with the development of the model. Hodgkinson in [6] found that the PSF of an idealized ocular fundus may be fitted using sech functions. Exponential functions has been also used to model scattering processes occurring in the eye $[23,24]$. In this work, we propose to use an exponential function to depict the response of the ocular fundus in the Fourier domain. In particular, we use a function of the form

$$
\operatorname{MTF}_{F}(u)=\exp \left(-2 \pi \frac{|u|}{r_{u}}\right)
$$

where $r_{u}$ may be interpreted as the correlation length of the scattering process in the frequency domain. We introduce the equality $r_{u}=1 / r_{x}$ to have an expression depending on correlation lengths given in object-space coordinates. Introducing equation 5 in the expression for the MTF given by equation 4 , we obtain the following,

$$
\operatorname{MTF}(u)=\operatorname{MTF}_{2}(u) M T F_{1}(u) \exp \left(-2 \pi r_{x}|u|\right)
$$

Last expression may be used to account for the effects of scattering in the DP process. The term $r_{x}$ that we call correlation length can be interpreted as an indirect estimation of the lateral spreading of light after the complex reflection process that occurs in the fundus. In the object-space domain, the response would be proportional the PSF of the ocular fundus,

$$
\operatorname{PSF}_{F}\left(x^{\prime \prime}\right) \propto \frac{1}{r_{x}^{2}+\left(m x^{\prime \prime}\right)^{2}}
$$

Following the work of Hodgkinson et al. in [6], the process in the choroid is dominated by forward scattering and the light returning towards the pupil comes from reflections in the boundaries of this layer. If it is true, variations of this parameter for a fixed wavelength may be related with the thickness of the choroid. The expression given in equations 6 and 7 may be used to predict the performance of the ocular fundus in the wavelength range going from around 600 to $800 \mathrm{~nm}$. Extrapolating the data presented in [6], we would expect a linear increment for $r_{x}$ within this range. Hence, the correlation length (lateral spreading) may be computed as

$$
r_{x}(\lambda)=r_{0}[1-s(800-\lambda)]
$$

where $r_{0}$ represents the correlation length evaluated at $800 \mathrm{~nm}, s$ is an scale factor, and $\lambda$ indicates the wavelength to be evaluated in nm. From the results presented in [6], we expect a slope for $r_{x}(\lambda)$ of around 0.45 between 600 and $800 \mathrm{~nm}$. Under such conditions, the scale factor takes a fixed value of $s=0.0028$. However, it would be necessary a characterization of the scattering at different wavelengths to provide better estimations of the parameter $s$.

Figure 1 presents the PSF of the ocular fundus for different wavelengths using the model given by equation 7 . The curves represent the response of the ocular fundus to incident light and they predict a widening in the PSF for longer wavelengths. This is a behavior expected in real situations based on the penetration properties of the fundus for different spectral components [8]. Based on this performance, we suggest that this model can be used to predict the scattering process occurring in the ocular fundus of the eye.

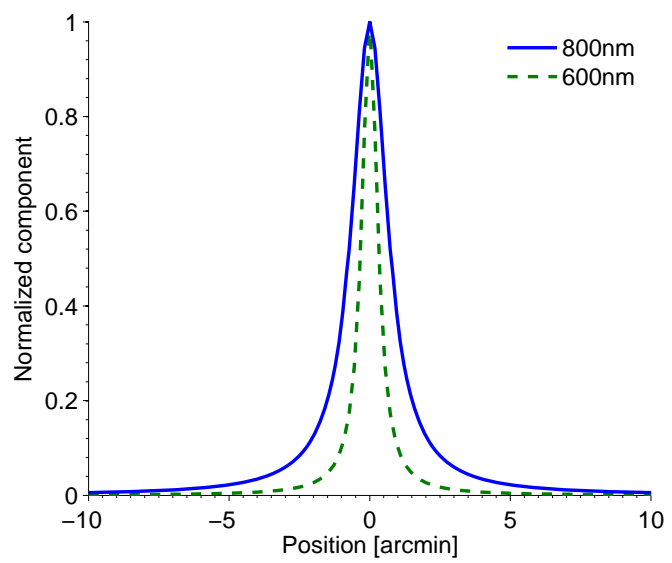

Fig. 1. PSF of the ocular fundus obtained for different wavelengths using the model for lateral spreading of light proposed in this work for $r_{0}=3.66 \mu \mathrm{m}$ and $s=0.0028$.

\section{Relationship between the DP and the HS MTF}

In HS sensors, the wavefront is sampled at the pupil plane using a lenslet array [2]. In this assessment mode, the information on the aberrations affecting the optics of the eye is estimated from variations in the position of the spots formed at the focal plane of the array. There is experimental evidence indicating that the position of the spot pattern results unaffected by the presence of scattering [3, 25]. Therefore, conventional HS data analysis provides information on aberrations, but not on scattering.

The expected behavior of the DP process without considering the influence of scattering can be estimated from HS data [4]. For instance, a synthetic DP MTF may be computed from the HS aberration map by multiplying the autocorrelations of the complex pupil functions [19] for the first and second pass pupil diameters $\left(M T F_{H S}=M T F_{1} \times M T F_{2}\right)$ 
[4]. The combination of this result with the full response of the DP process $\left(M T F_{D P}\right)$ given by equation 4 suggests that the ratio of the DP to the HS MTF $\left(M T F_{R t}\right)$ may be used to estimate, until the cut-off frequency of the HS response, the effects of the scattering produced in the ocular fundus as,

$$
\operatorname{MTF}_{R t}(u)=\frac{M T F_{D P}(u)}{M T F_{H S}(u)}=\operatorname{MTF}_{F}(u)
$$

However, last expression is valid only when there is not any other scattering than the one produced by the interaction of light with the ocular fundus and when wave aberrations affecting the eye are well described by HS data. Equation 9 should be modified to include the effects of intraocular scattering. In addition, changes in intensity observed at the pupil plane due to the waveguide properties of photoreceptors (Stiles-Crawford effect) [7] have not been considered during the development of the model.

\section{RESPONSE FOR SCATTERING IN YOUNG HEALTHY EYES}

\section{A. Apparatus}

Figure 2 shows an schematic diagram of the apparatus that was used to measure the scattering properties of the ocular fundus in young healthy eyes. The binocular system is able to provide comparable DP and HS optical quality estimators. The characteristics, calibration process, and validation of the instrument are described in detail elsewhere [12].

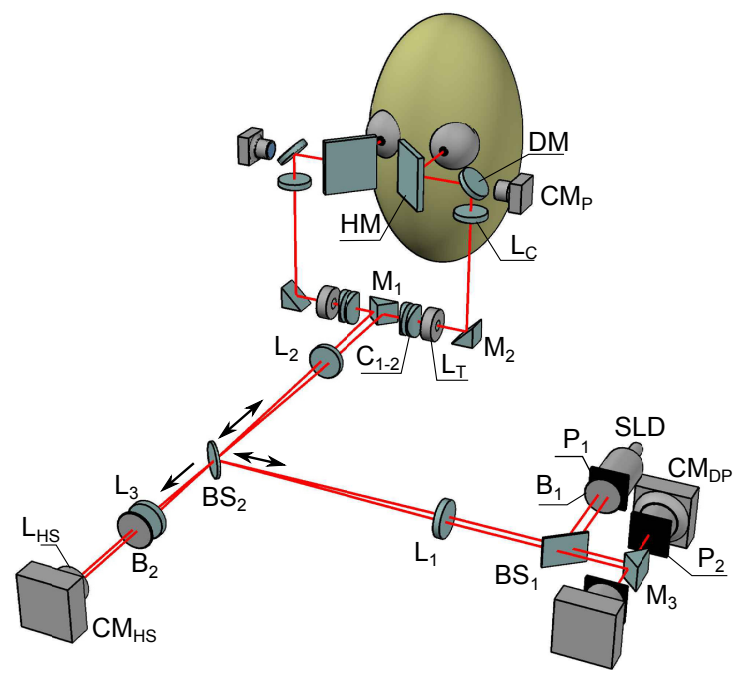

Fig. 2. Representation of the system used during experimentation with labels for elements belonging to the left-eye optical path. Light source: $S L D$; apertures: $P_{1}, P_{2}$; polarizers: $B_{1}, B_{2}$; beam splitters: $B S_{1}, B S_{2}$; lenses: $L_{1}, L_{2}, L_{3}$; mirrors: $M_{1}, M_{2}, M_{3}$; tunable devices: $C_{1-2}, L_{T}-L_{C}$; dichroic mirror: $D M$; hot mirror: $H M$; lenslet array: $L_{H S}$; cameras: $C M_{P}, C M_{D P}, C M_{H S}$.

During measurements, light coming from the superluminescent diode $S L D$ with a peak emission of $801 \mathrm{~nm}$ and a power spectrum of $37 \mathrm{~nm}$ is focused on the ocular fundus of both eyes. Then, a pair of CMOS cameras $C M_{D P}$ is used to record the aerial retinal images of the left and right eyes, respectively, with a pixel resolution of $0.182 \mathrm{arcmin}$.
The entrance and exit pupils $P_{1}$ and $P_{2}$ of 2 and $4 \mathrm{~mm}$ in diameter, respectively, permit having a DP system working in an asymmetric configuration [17]. The lenses forming the telescopic system $L_{1}-L_{2}$ are identical in optical power and they impose a magnification of $|m|=1$ to the DP instrument. In the case of the HS sensor, the single micro-lens array $L_{H S}$ of $200 \mu \mathrm{m}$ in pitch and $6.3 \mathrm{~mm}$ in focal length, followed by the CMOS camera $C M_{H S}$, is used to sample the wavefront of both eyes at a plane conjugate to the pupil of the eye. The magnification of $|m|=0.5$ imposed by the telescopic system $L_{2}-L_{3}$ allows to use a single HS sensor to assess both eyes simultaneously. The impact of corneal reflections in HS images is diminished by using the set of crossed polarizers $B_{1}$ and $B_{2}$ positioned within the first and second pass of light, respectively.

The system permits evaluations of both eyes in normal viewing conditions while subjects look at external targets through a pair of hot mirrors HM. Experimental measurements of the incident power indicate that the left and right eyes are exposed to 0.76 and $0.78 \mu \mathrm{W}$ during the measurements. A set of dichroic mirrors $D M$ and cameras $C M_{P}$ allows to monitor the pupil position for alignment purposes. Changes in the orientation between the cylindrical lenses forming the set $C_{1-2}$ permits compensations for astigmatism of the eyes under assessment [26]. The focus tunable lens $L_{T}$ is used in combination with the lens of fixed optical power $L_{C}$ to compensate for defocus [27]. The prism mirrors $M_{1}$ and $M_{3}$ are used to separate or join the optical path of the light going to or coming from the left and right eyes. While beam splitter $B S_{1}$ enables to separate the first and second pass of light in the DP instrument, $B S_{2}$ splits the light going to the DP and HS sensor.

\section{B. Experimental procedure}

The DP and the HS MTF were obtained for the left eye of 8 young healthy subjects using the left-eye optical path of a calibrated version of the system described above. The subjects were $26 \pm 4$ years old in average and standard deviation and presented dark-brown eyes with clear optical media. The task of the subjects was to fixate in normal visual conditions an external target located at a distance of 6 meters away. After properly setting up the power of the laser source, 5 series of $20 \mathrm{DP}$ and HS images were recorded for each left eye of the 8 subjects while they were compensated for low-order aberrations (astigmatism and defocus). In this manner, the refractive errors remained below $0.25 \mathrm{D}$ during measurements. All the images were recorded using an integration time of $40 \mathrm{~ms}$. For each subject, the measured data was obtained in a single session of less than $10 \mathrm{~min}$ in duration under similar environment illumination conditions.

\section{Data processing}

The averages of the 20 images composing the series were used to compute MTF curves. In the case of DP data, the resultant image was Fourier transformed. Then, the MTF was obtained after proper normalization by taking the radial profile of the spectral representation and applying a peak correction [28] by extrapolating lower frequencies from a curve fitting based on exponential functions [29]. When working with HS data, the wave aberration was estimated using 54 Zernike coefficients. Then, the MTF was computed as the autocorrelation of the complex pupil functions [19] for the first and second pass pupil diameters. Both the DP and the HS data were compensated for residual errors attributed to the measuring system by following the procedure described in [12]. 
At the end of the process described above, $5 \mathrm{DP}$ and 5 HS estimations were available per subject. After this, the averages of the 5 estimations were used to compute the ratio $M T F_{R t}=M T F_{D P} / M T F_{H S}$. Posteriorly, $M T F_{R t}$ was fitted to the model described by equations 5 and 6 . In this manner, the resultant values of correlation lengths $r_{x}$ correspond to the ones producing the best coincidences between $M T F_{R t}$ and $M T F_{F}$ at the working wavelength. The computations were performed using a nonlinear least squares method programmed in Matlab (MathWorks, 2010). Additionally, the fitting process considered only those points for which the $M T F_{D P}$ and $M T F_{H S}$ presented values above 0.15 . Therefore, the model excluded the part of the curves around which the ratio may become indeterminate.

Besides the MTF, there are other parameters that were obtained for each of the images belonging to a series. In the object domain, the average intensity at the edges of the raw DP images was obtained. This parameter is denoted as $I_{e}$ and was estimated by considering all those pixels whose round distance from center were higher than 120 using cropped versions of the images of $256 \times 256$ pixels in size. In addition, the objective scatter index (OSI) [4] was obtained from the DP images as an estimator of intraocular scattering. This parameter was computed as OSI $=0.1\left(I_{R} / I_{0}\right)$, were $I_{R}$ and $I_{0}$ represent the integrated intensity contained in a ring between 12 and 20arcmin and that contained in the central part of the image for angles lower than 1arcmin, respectively. On the other hand, the Strehl ratio was obtained for both the DP and the HS data. This parameter was estimated as the area under the unidimensional MTF. Because the evaluation of the scattering process is based on differences between DP and HS estimations, we computed the ratio between Strehl ratios $S R_{R t}=S R_{D P} / S R_{H S}$ for each series of measurements. As a result of these computations, 5 estimations of the $M T F_{D P}, M T F_{H S}, I_{e}, O S I$, and $S R_{R t}$ were available per assessed eye. However, the parameters presented in this work refer to the average values of those estimations. In this manner, we provide a single curve or value containing the contribution of all the series of recordings for each subject.

\section{Results and discussion}

The PSF for both the DP and HS techniques presented similar behaviors in all the measured cases. Figure 3 depicts the performance for 3 subjects. The cases are labeled as FA, IC, and SE. Visual inspection of the images demonstrate the similarities in terms of shape between DP and HS estimations. For instance, the characteristic aspect observed for subject $\mathrm{SE}$ is distinguished in the images regardless the technique. Another example of the similarities is observed in images for subject IC. There, a diamond-like shape is observed in both the DP and HS estimations. Although the aspect of the images follows similar patterns, it seems that DP cases include a veil surrounding the central part of the spot. This situation induces contours in the DP images less defined than in the HS estimations. Such characteristic may arise from angular deviation of light due to the presence of scattering in the DP process.

The deviations between DP and HS data can be noticed in figure 4 . Beside the average curve, the individual estimations for $M T F_{R t}$ are plotted in the figure. As observed, the curves follow a decreasing tendency around lower frequencies. Then, they present a constant behavior until reaching the cutoff frequency, were the ratio become indeterminate. There is a variability among subjects in the slope of the response in the first part of the spectrum. In general, the constant magnitude in the central part of the spectrum decreases with

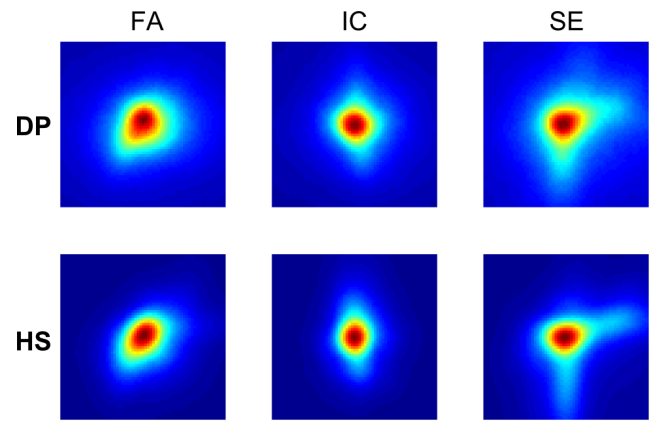

Fig. 3. $64 \times 64$ pixels cropped versions of the PSF from DP (top) and HS (bottom) data for 3 different subjects.

increments of the slope. However, this behavior may be attributed more to the decrement in the magnitude of the MTF around those frequencies than to variations of any ocular or scattering condition among assessed eyes. This fact should be considered by giving more weight to lower frequencies during the estimation of any other parameter when using the ratio $M T F_{R t}$. For this reason, the fitting process considered only the part of the normalized DP and HS spectrum with magnitudes above 0.15 . Under such condition, the application of the model indicates that the $M T F_{R t}$ curves for the 8 measured subjects may be fitted by $M T F_{F}$ using a correlation length of $r_{x}=$ $3.66 \pm 0.50 \mu \mathrm{m}$ in mean and standard deviation.

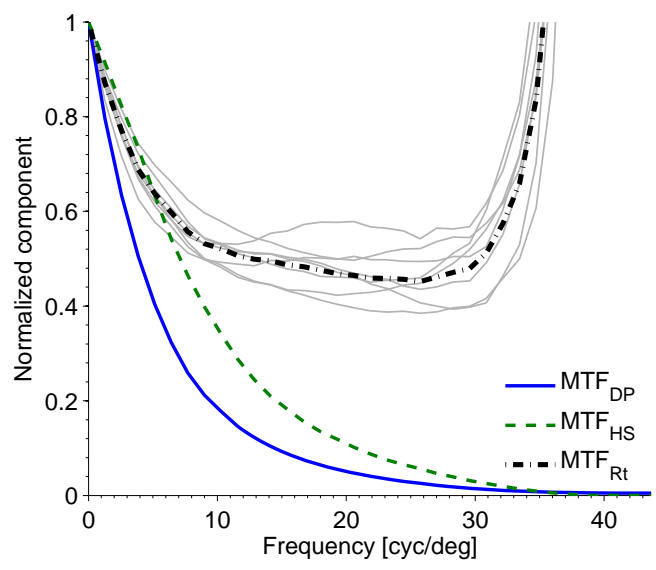

Fig. 4. Average DP (solid line) and HS (dashed line) MTF for the 8 subjects. The average $M T F_{R t}$ is plotted in the figure (black dashed-dotted line), as well as their individual estimations (gray solid lines).

Light scattered in the ocular fundus was considered as the main source of deviations between DP and HS data during the measurements. The subjects presented an OSI of $0.46 \pm 0.16$ in mean and standard deviation with a maximum value of 0.67 . These values are in agreement to those observed in normal eyes (without cataracts) [4]. Based on the small OSI values and on the visual inspection of the ocular media performed during the measurements, the impact of the intraocular scattering on the acquired data can be considered as negligible.

Figure 5 depicts the performance of the HS response after including the effects of light scattered in the ocular fundus. To obtain the curves, the scattering process was included into the computed data by multiplying the HS responses by $M T F_{F}$ for 


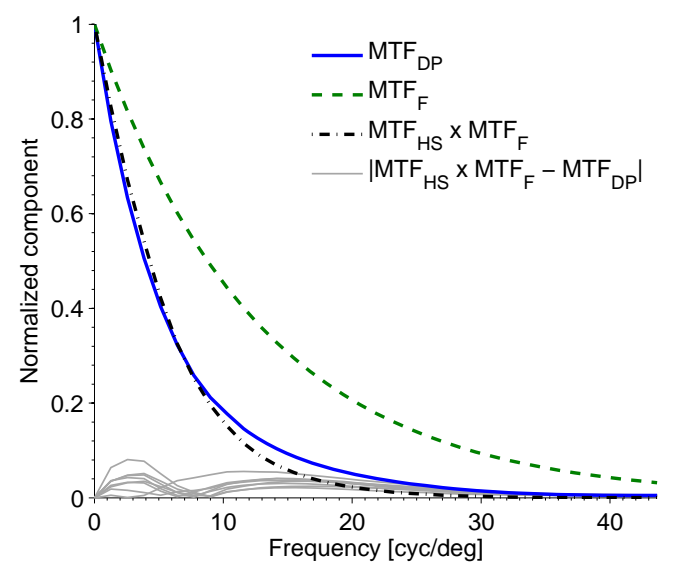

Fig. 5. Comparison between the average $M T F_{D P}$ (solid line), $M T F_{F}$ (dashed line), and $M T F_{H S} \times M T F_{F}$ (dashed-dotted line). The modulus of the absolute error between $M T F_{H S} \times M T F_{F}$ and $M T F_{D P}$ is also plotted, but using the individual DP and HS estimations for the 8 subjects (gray solid lines).

$r_{x}=3.66 \mu \mathrm{m}$. The model was applied not only to the average curve $M T F_{H S}$, but also to the individual HS estimations of the 8 subjects. Considering the average curves, it is observed that the HS data describe well the behavior of $M T F_{D P}$ when the model for scattering is included in the computations. The inclusion of $M T F_{F}$ into the individual estimations indicates that the HS response predicts the DP behavior with a peak absolute deviation of around 0.08 and an average root mean square (RMS) error of 0.02 for frequencies below $43.63 \mathrm{cyc} / \mathrm{deg}$. It has to be mentioned that the latter error is around 5 times lower than 0.11 , the average RMS deviation between the DP and HS responses before including the effects of scattering.

The parameter $r_{x}$ denotes the lateral spreading of light provoked by the scattering process. The average performance of the measured subjects suggests that each component of the incident light is spread in average $3.66 \mu \mathrm{m}$ after interacting with the ocular fundus. The PSF of this process would correspond to the one depicted in figure 1. Some authors have suggested that the interaction of light with the ocular fundus is dominated by forward scattering and the light that travels from layers beyond the retina towards the pupil comes from reflections at the boundaries of the choroid [6]. If it is true, $r_{x}$ may be also considered an indirect estimator of the thickness of this layer because the amount of lateral spreading increases as it penetrates into the fundus. However, it is necessary to perform measurements in light-colored eyes to estimate the influence of the content of melanin in the choroid on the computed parameter.

\section{E. Relationship between the lateral spreading of light and other effects of scattering in DP images}

The lateral spreading of light provided by the model is presented in figure 6 in function of the mean intensity observed at the edge of the DP images. In overall terms, a proportional relationship is observed between these two parameters among measured data. During DP measurements and discarding scattering from the lens of the eye, the parameter $I_{e}$ could vary by changes in the environment light or in back-reflections in the optics of either the instrument or the eye. However, the measurements were performed under the same dark conditions and we did not perceive any back-reflected light that could compromise the computation of $I_{e}$. Although variations in the power of the laser source could be another factor influencing the intensity at the edge of the images, it was not necessary to modify this parameter during measurements. Assuming an equivalent ocular transmittance among subjects, we suggest that variations of $I_{e}$ among the experimental data are leaded mainly by scattering in the ocular fundus.

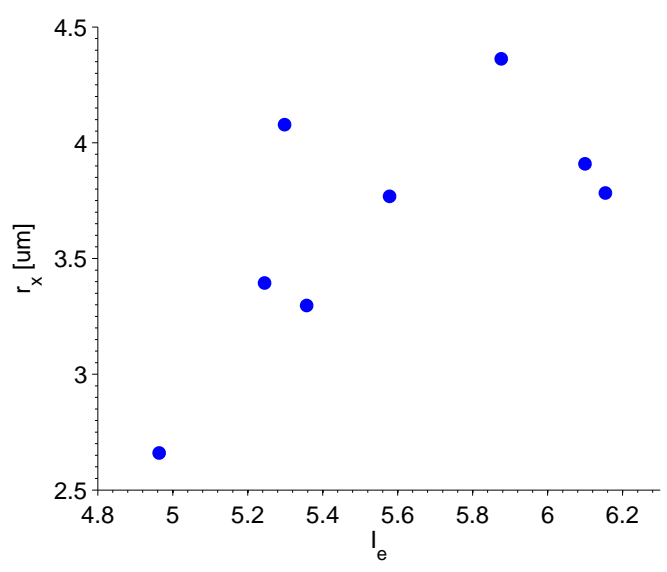

Fig. 6. Lateral spreading of light $r_{x}$ in function of the mean intensity at the edge of double-pass images $I_{e}$.

The agreement between $r_{x}$ and $I_{e}$ indicates that the constant background noise observed in DP images may be predicted using the model for lateral spreading of light provided by the model presented in this work. This situation suggests that $r_{x}$ could be used to improve the accuracy of parameters in which the background noise is considered at wavelengths affected by scattering from deeper layers of the ocular fundus. For instance, the uncertainty during the classification of cataracts at different wavelengths using the OSI may be reduced by introducing somehow the lateral spreading of light to the computations.

Figure 7 depicts the relationship between $r_{x}$ and the ratio $S R_{R t}$. Since larger values of lateral spreading of light indicates higher deviations of DP response with respect HS data, the inverse relationship between these two parameters was expected. Although they both vary in function of $r_{x}$, the light at the edges of the DP images $I_{e}$ and the ratio between the DP and HS Strehl ratios $S R_{R t}$ are quantities that are not directly related with each other. The effects of the constant background noise provided by $I_{e}$ are observed in the Fourier domain as an increment of the response at zero frequency, which is then compensated by the peak correction during the computation of the MTF [28]. Although the results should be corroborated using a bigger population, the trends observed in the plots of figures 6 and 7 provide information on the same phenomena, but measured in different manners. In addition, the influence of the peak correction process and of higher order aberrations undetected by the HS sensor on the computed parameters should be studied.

\section{CONCLUSIONS}

This work presented an analysis on the scattering properties of the ocular fundus based on differences between DP and HS measurement. To do this, we used a system that provides comparable data using these two techniques and works in 


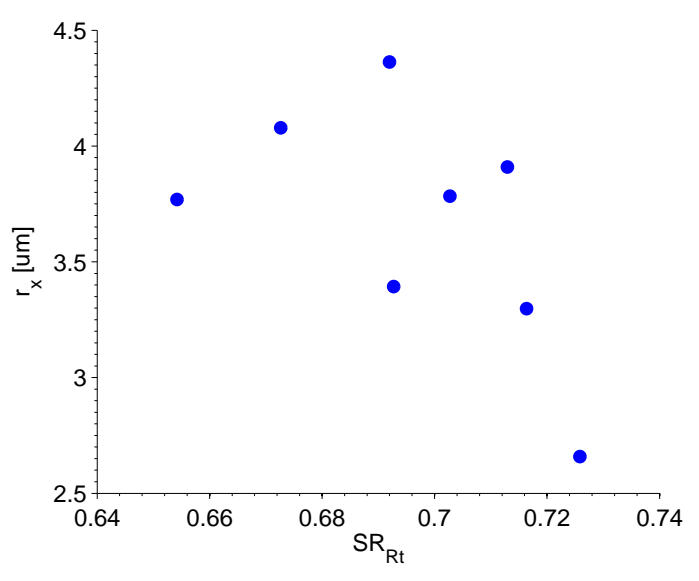

Fig. 7. Lateral spreading of light $r_{x}$ in function of the ratio between the DP and HS Strehl ratios $S R_{R t}$.

infrared light. A model accounting for the differences between techniques has been introduced. Such a model is based on parameters that provide information of the lateral spreading of light produced by the ocular fundus. The experimental results suggest that the model proposed here fit well the differences between DP and HS measurements considering only those frequencies at which the MTF contains enough information on the processes occurring in the eye. The performance of fundal scattering should be characterized in a more accurate manner by determining with objective (or subjective) methods the influence of the eye lenses during measurements. However, we suggest that the tendencies presented in this work reflect the influence of fundal scattering in DP measurements of darkbrown eyes.

The characterization of the scattering process in the ocular fundus may be used not only to understand the differences between DP and HS data, but also to improve the accuracy of other parameters. For instance, the background noise affecting DP images influences the computation of the Objective Scatter Index, which has been used to classify cataracts. However, the results suggest that this background noise may be modified with variations of fundal scattering.

The results presented here show that the ocular scattering may affect the computation of the Strehl ratio, which is a parameter usually used to provide information on the retinal image quality. We cannot assure that the scattering in the ocular fundus at visible light affects the perception of external scenes during the image formation process. However, the deviation from average values of the parameters with information on this type of scattering may be an indicator of other problems that could be related at some point with vision. Nevertheless, a further research is necessary to corroborate this point.

\section{FUNDING}

Ministerio de Economía y Competitividad (MINECO) (DPI2014-56850-R); European Community; Education, Audiovisual and Culture Executive Agency (EACEA) (EMJDEurophotonics).

\section{REFERENCES}

1. J. Santamaría, P. Artal, and L. Bescós, "Determination of the pointspread function of human eyes using a hybrid optical-digital method,"
J. Opt. Soc. Am. A 4, 1109-1114 (1987).

2. J. Liang, B. Grimm, S. Goelz, and J. F. Bille, "Objective measurement of wave aberrations of the human eye with the use of a Hartmann-Shack wave-front sensor," J Opt. Soc. Am. A 11, 1949-1957 (1994).

3. F. Díaz-Doutón, A. Benito, J. Pujol, M. Arjona, J. L. Güell, and P. Artal, "Comparison of the Retinal Image Quality with a Hartmann-Shack Wavefront Sensor and a Double-Pass Instrument," IOVS 47, 1710-1726 (2006).

4. P. Artal, A. Benito, G. M. Pérez, E. Alcón, A. de Casas, J. Pujol, and J. M. Marín, "An objective scatter index based on double-pass retinal images of a point source to classify cataracts," PLoS ONE 6, e16823 (2011).

5. D. R. William, D. H. Brainard, M. J. McMahon, and R. Navarro, "Doublepass and interferometric measures of the optical quality of the eye," $\mathrm{J}$. Opt. Soc. Am. A 11, 3123-3435 (1994).

6. I. J. Hodgkinson, A. C. B. Molteno, and P. B. Greer, "Point-spread function for light scattered in the human ocular fundus," J. Opt. Soc. Am. A 11, 479-486 (1994).

7. S. Marcos, S. Burns, and J. C. He, "Model for cone directionality reflectometric measurements based on scattering," J. Opt. Soc. Am. A 8, 2012-2022 (1998).

8. H. S. Ginis, G. M. Pérez, J. M. Bueno, A. Pennos, and P. Artal, "Wavelength dependence of the ocular strylight," IOVS 54, 3702-3708 (2013).

9. F. C. Delori and K. P. Pflibsen, "Spectral reflectance of the human ocular fundus," Applied Optics 28, 1061-1077 (1989).

10. J. Liang and D. R. Williams, "Aberrations and retinal image quality of the normal human eye," J. Opt. Soc. Am. A 14, 2873-2883 (1997).

11. P. Artal and R. Navarro, "Simultaneous measurement of two-pointspread functions at different locations across the human fovea," Applied Optics 39, 3646-3656 (1992).

12. C. E. García-Guerra, M. Aldaba, M. Arjona., and J. Pujol, "Binocular open-view system to perform estimations of aberrations and scattering in the human eye," Applied Optics 54, 9504-9508 (2015).

13. N. López-Gil and P. Artal, "Comparison of double-pass estimates of the retinal-image quality obtained with green and near-infrared light," $\mathrm{J}$. Opt. Soc. Am. A 14, 961-971 (1997).

14. M. Vilaseca, A. Padilla, J. C. Ondategui, M. Arjona, J. L. Güell, and J. Pujol, "Effect of laser in situ keratomileusis on vision analyzed using preoperative optical quality," Journal of Cataract and Refractive Surgery 36, 1945-1953 (2010).

15. M. Aldaba, M. Vilaseca, F. Díaz-Doutón, M. Arjona, and J. Pujol, "Measuring the accommodative response with a double-pass system: Comparison with the Hartmann-Shack technique," Vision 62, $26-34$ (2012).

16. J. A. Martínez-Roda, M. Vilaseca, J. C. Ondategui, A. Ginner, F. J. Burgos, G. Cardona, and J. Pujol, "Optical quality and intraocular scattering in a healthy young population," Clinical and Experimental Optometry 94, 223-229 (2010).

17. P. Artal, S. Marcos, R. Navarro, and D. R. Williams, "Odd aberrations and double-pass measurements of retinal image quality," J Opt. Soc. Am. A 12, 195-201 (1995).

18. L. Diaz-Santana and J. C. Dainty, "Effects of retinal scattering in the ocular double-pass process," J. Opt. Soc. Am. A 18, 1437-1444 (2001).

19. J. W. Goodman, Introduction to Fourier Optics (McGraw-Hill, 1998), 2nd ed.

20. P. Beckmann, "Scattering by composite rough surfaces," Proceedings of the IEEE 53, 1012 - 1015 (1965).

21. J. C. Dainty, "The statistics of speckle patterns," in "Progress in Optics XIV," , E. Wolf, ed. (1976), pp. 1-46.

22. S. Haykin and B. V. Veen, Signals and Systems (Wiley, 2002), 2nd ed.

23. P. Rodríguez and R. Navarro, "Double-pass versus aberrometric modulation transfer function in green light," Journal of Biomedical Optics 12, 044018 (2007).

24. R. Navarro, J. A. Méndez-Morales, and J. Santamaría, "Optical quality of the eye lens surfaces from roughness and diffusion measurements,' J. Opt. Soc. Am. A 3, 228-234 (1986).

25. M. J. Cox, D. A. Atchison, and D. H. Scott, "Scatter and its 
implications for the measurement of optical image quality in human eyes," Optometry and Vision Science 80, 58-68 (2003).

26. J. Arines and E. Acosta, "Low-cost adaptive astigmatism compensator for improvement of eye fundus camera," Optics Letters 36, 4164-4166 (2011).

27. F. Sanàbria, F. Díaz-Doutón, M. Aldaba, and J.Pujol., "Spherical refractive correction with an electro-optical liquid lens in a double-pass system," J. Europ. Opt. Soc. Rap. Public. 8, 13062 (2013).

28. P. Artal, I. Iglesias, N. López-Gil, and D. G. Green, "Double-pass measurements of the retinal-image quality with unequal entrance and exit pupil sizes and the reversibility of the eye's optical system," J. Opt. Soc. Am. A 12, 2358-2366 (1995).

29. P. Artal and R. Navarro, "Monochromatic modulation transfer function of the human eye for different pupil diameters: an analytical expression," J. Opt. Soc. Am. A 11, 246-249 (1994). 